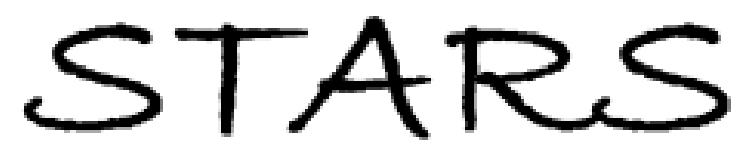

University of Central Florida

STARS

$1-1-2010$

\title{
Droplet actuation on a liquid layer due to thermocapillary motion: Shape effect
}

Ehsan Yakhshi-Tafti

University of Central Florida

Hyoung J. Cho

University of Central Florida

Ranganathan Kumar

University of Central Florida

Find similar works at: https://stars.library.ucf.edu/facultybib2010

University of Central Florida Libraries http://library.ucf.edu

This Article is brought to you for free and open access by the Faculty Bibliography at STARS. It has been accepted for inclusion in Faculty Bibliography 2010 s by an authorized administrator of STARS. For more information, please contact STARS@ucf.edu.

\section{Recommended Citation}

Yakhshi-Tafti, Ehsan; Cho, Hyoung J.; and Kumar, Ranganathan, "Droplet actuation on a liquid layer due to thermocapillary motion: Shape effect" (2010). Faculty Bibliography 2010s. 962.

https://stars.library.ucf.edu/facultybib2010/962

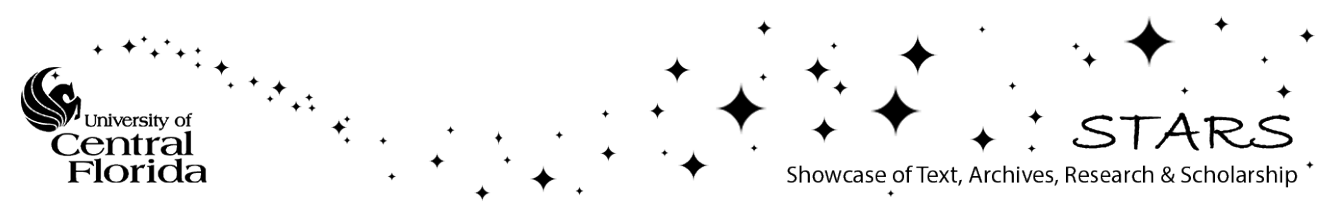




\section{Droplet actuation on a liquid layer due to thermocapillary motion: Shape effect}

Cite as: Appl. Phys. Lett. 96, 264101 (2010); https://doi.org/10.1063/1.3456391

Submitted: 13 February 2010 . Accepted: 28 May 2010 . Published Online: 30 June 2010

Ehsan Yakhshi-Tafti, Hyoung J. Cho, and Ranganathan Kumar

\section{ARTICLES YOU MAY BE INTERESTED IN}

Thermocapillary migration of interfacial droplets

Physics of Fluids 21, 042105 (2009); https://doi.org/10.1063/1.3112777

Thermocapillary actuation of binary drops on solid surfaces

Applied Physics Letters 99, 104101 (2011); https://doi.org/10.1063/1.3632041

Thermocapillary migration of an attached drop on a solid surface

Physics of Fluids 6, 3183 (1994); https://doi.org/10.1063/1.868096

\section{Applied Physics Reviews} Now accepting original research 


\title{
Droplet actuation on a liquid layer due to thermocapillary motion: Shape effect
}

\author{
Ehsan Yakhshi-Tafti, ${ }^{1}$ Hyoung J. Cho, ${ }^{1,2}$ and Ranganathan Kumar ${ }^{1, a)}$ \\ ${ }^{1}$ University of Central Florida, Orlando, Florida 32816, USA \\ ${ }^{2}$ Sungkyunkwan University, Jangan-Gu, Suwon, Gyeonggi-Do 440-746, Korea
}

(Received 13 February 2010; accepted 28 May 2010; published online 30 June 2010)

\begin{abstract}
In the thermocapillary migration of droplets on the free surface of immiscible liquids, we observe that the lens-shaped drops move from warm toward cooler regions while spherical drops move in the opposite direction. We explain this dual behavior using an analysis of surface deformation and velocity profiles of thin liquid layers subject to a lateral thermal gradient. Liquid platforms allow thermocapillary transport of drops with higher migration speeds than solid substrates and lower internal temperature fluctuation. Such conditions may be exploited in biochemical microsystems where droplet evaporation, contamination, and surface pinning need to be avoided. (C) 2010 American Institute of Physics. [doi:10.1063/1.3456391]
\end{abstract}

Chemically patterned solid substrates with integrated heaters have been used to actuate droplets ${ }^{1}$ and a pumping mechanism for droplets based on this concept has been proposed. ${ }^{2}$ Most studies and applications of thermocapillary migration of droplets have been reported for drops resting on the surface of nonwettable solid substrates. ${ }^{1,34}$ Drawbacks such as pinning of droplets with sizes below a critical limit to the surface, low migration speeds (approximately millimeter per minute), direct exposure of the drop contents to hot surfaces leading to evaporative losses, ${ }^{1}$ and irreversibility of motion due to hysteresis, make wetted surfaces and liquid carrier platforms an attractive alternative in light of practical applications such as droplet-based biochemical systems. ${ }^{5-7}$ In most studies of drops on solid surfaces, ${ }^{1,3,4}$ drops were seen to move toward the colder end of the thermal gradient. We show through experiments and analysis that drops of the same volume, depending on their shape at the free interface, can be propelled in either direction of the temperature gradient.

Figure 1 shows a drop of water which can be dispensed in either spherical (3.2 $\mathrm{mm}$ diameter) or lens-shape (2 mm equivalent diameter) configurations on a thin liquid layer of fluorocarbon (FC-43) used as the carrier liquid platform (depth, $d=2 \mathrm{~mm}$, length, $l=55 \mathrm{~mm}$, width, $w=8.5 \mathrm{~mm}$, and aspect ratio $\mathrm{AR}=1 / 28)$. We show that spherical drops above the interface can be formed by releasing pendant drops from a critical height onto the target pool. Noncoalescent spherical drops on the surface of similar liquids are attributed to trapped air which serves as a lubricating film. ${ }^{8,9}$ The physical properties and the interfacial gradients are provided in Table I. When the thermal gradient is maintained, the quiescent thin layer of liquid is set in motion, which develops into a larger circulation flow over the whole liquid layer. In the close-up view of 8-10 $\mathrm{mm}$ near the heater (Fig. 2), the deformation of the liquid free surface due to the tangential stresses is seen, with maximum depression in height near the heater on the left. The deformed free surface profile is the result of the balance between thermocapillary stress due to the thermal gradient and viscous stresses due to the developed flow. Since the surface (interfacial) tension decreases

${ }^{a)}$ Electronic mail: rnkumar@mail.ucf.edu. with increase in temperature, the fluid motion is from the hot region to the cold near the surface. The attraction (to the heater) of the spherical drop and the repulsion mode of the lens are recorded by a video camera and the thermal field generated on the surface and the drops is visualized using a high resolution infrared (IR) camera.

Motion of the lens-shaped drop. The measured IR temperature profile follows the exponential solution of heat transfer in a semi-infinite slab with a constant heat source at the boundary. The direction of thermocapillary migration for the lens-shaped drop is toward the lower temperature gradient as found in other papers, i.e., ${ }^{1,4,7,10}$ drops moved from warm to colder regions. A $4 \mu \mathrm{l}$ lens-shaped drop (equivalent diameter $2 \mathrm{~mm}$ ) is subjected to two different gradients and its velocity and temperature variation are provided in Fig. 3 (Video 1). The IR panels show that the drop gets repelled from the heat source and carried away in the same direction of the liquid due to large contact area and viscous drag. If the drop were to be placed on a solid surface without a carrier liquid, the thermocapillary motion would be determined by the balance of the internal flows within the drop (Marangoni flow) together with the change in the spreading coefficient $\left(\mathrm{S}=\sigma_{\text {carrier }}-\sigma_{\text {carrier drop }}-\sigma_{\text {drop }}\right)$ at the interface at the extremities of the droplet. ${ }^{3}$ However, when it is dispensed on a liquid platform near the heater, it reaches the surface velocity and thereafter moves from warm to colder regions under the influence of the surface flow. The drop velocity can be as high as $4 \mathrm{~mm} / \mathrm{s}$ at higher gradients [Fig. 3(d)]. Highest speeds achieved on solid surfaces are an order of magnitude lower than the droplet speeds we measured on a liquid sur-

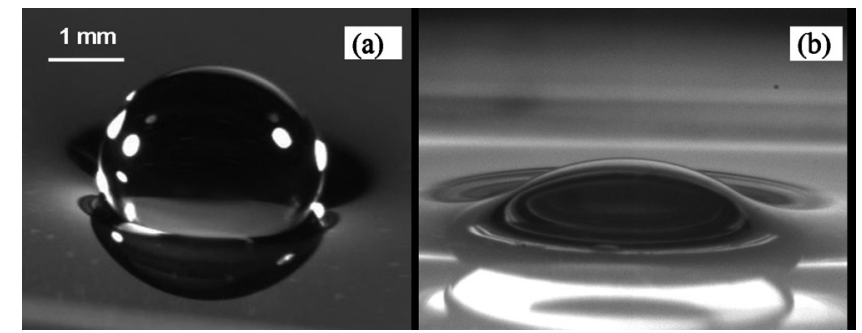

FIG. 1. Aqueous drops resting on the free surface of fluorocarbon liquid (FC-43) in a spherical mode (a) and lens mode (b). 
TABLE I. Physical properties of aqueous droplet and the fluorocarbon carrier liquid.

\begin{tabular}{|c|c|c|c|c|c|c|}
\hline Fluid & $\begin{array}{c}\rho \\
\left(\mathrm{g} / \mathrm{cm}^{3}\right)\end{array}$ & $\frac{\nu}{(\text { centiStoke) }}$ & $\begin{array}{c}k \\
\left(\mathrm{~W} / \mathrm{m}^{\circ} \mathrm{C}\right)\end{array}$ & $\begin{array}{c}\beta \\
{\left[\times 10^{-3}\right.} \\
\left.\left(1 /{ }^{\circ} \mathrm{C}\right)\right]\end{array}$ & $\begin{array}{c}\sigma \\
{\left[\times 10^{-3}\right.} \\
(\mathrm{N} / \mathrm{m})]\end{array}$ & $\begin{array}{c}d \sigma / d T \\
{\left[\times 10^{-3}\right.} \\
\left.(\mathrm{N} / \mathrm{m}) /{ }^{\circ} \mathrm{C}\right]\end{array}$ \\
\hline FC-43 & 1.88 & 2.8 & 0.066 & 1.2 & 16 & -0.099 \\
\hline Water & 1 & 1 & 0.58 & 0.21 & 71 & -0.20 \\
\hline FC-43/Water & & & & & 52 & -0.14 \\
\hline
\end{tabular}

face for similar values of thermal gradient. The liquid surface temperature varied by $\sim 15{ }^{\circ} \mathrm{C}$ over the length of the channel for the higher gradient. However, the maximum temperature increase on the droplet surface was only $5{ }^{\circ} \mathrm{C}$ [Fig. 3(c)]. Limited temperature fluctuation is critical in biochemical applications; reasonable transport speeds are observed for transported drops $\left(<5{ }^{\circ} \mathrm{C}\right)$ on liquid platforms. Partially submerged lens shaped drops consistently moved from hot to cold regions, however, drop velocities were lower for larger drops (not shown). This observation is contrary to those made on solid substrates where larger drops achieved relatively higher velocities ${ }^{4}$ since smaller sized drops have a greater tendency to get pinned to the surface.

Motion of the spherical drop. When drops are dispensed onto the surface of other immiscible liquids, partially submerged lens shaped drops are usually formed at the interface. We found that it is also possible to generate isolated spherical drops resting above the free surface by releasing them from a critical height range. Interestingly, drops with such spherical configurations travel in the opposite direction to the commonly observed lens shaped drops discussed earlier. Figure 4 and Video 2 show a spherical drop $(17 \mu$ l; diameter $3.2 \mathrm{~mm}$ ) migrate toward the heater from a distance of $40 \mathrm{~mm}$ apart. The drop speeds up as it approaches the heater where the interface slope is maximum. Thermally induced deformation of thinner a liquid layer of $2 \mathrm{~mm}$ is more pronounced (Fig. 2) due to surface tension variation compared to thicker ones.

The governing equations are as follows: ${ }^{11,12}$

$$
\begin{aligned}
& \left(d^{2} / v t\right) \frac{\partial u}{\partial t}=-\frac{\partial p}{\partial x}-\mathrm{Bo} \cdot \mathrm{AR}^{2} \frac{\partial h}{\partial x}+\frac{\partial^{2} u}{\partial y^{2}}, \\
& \frac{\partial p}{\partial y}=0
\end{aligned}
$$
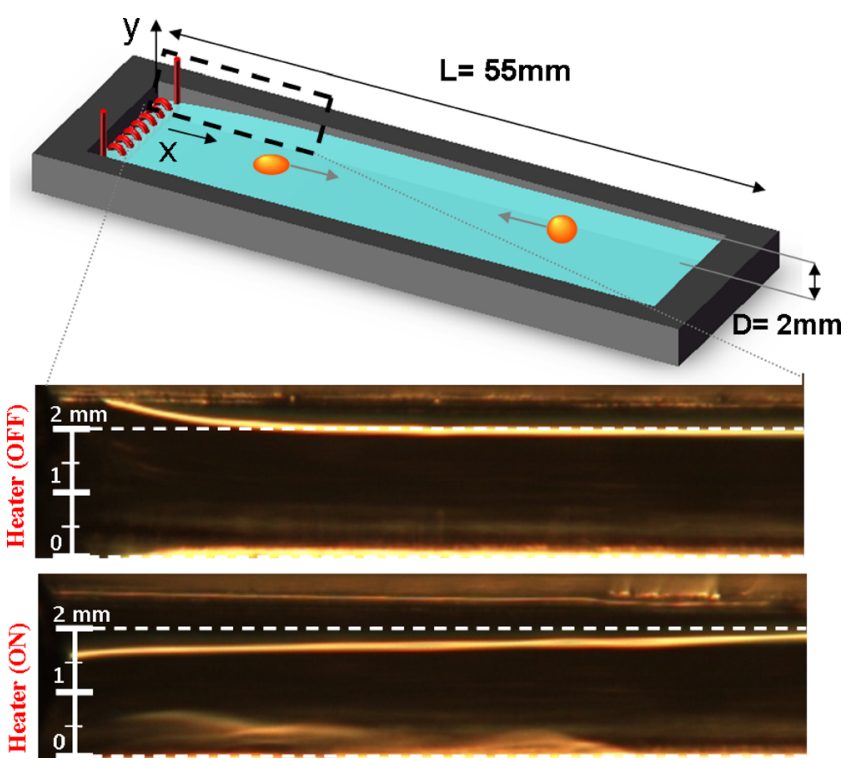

FIG. 2. (Color online) Schematic of the experimental setup (top). Photographs of initial surfaces of the liquid layer and when the heater is turned on (bottom). (Rise at the top left corner when heater is off, is due to the capillary rise of the meniscus at the solid wall; dashed line shows the undeformed free surface.)

$$
\left(d^{2} p r / v \tau\right) \frac{\partial \theta}{\partial t}+\mathrm{Ma} \cdot \mathrm{AR}^{2}\left(u \frac{\partial \theta}{\partial x}+v \frac{\partial \theta}{\partial y}\right)=\frac{\partial^{2} \theta}{\partial^{2} y},
$$

with initial conditions, $t=0 ; u=0 ; v=0 ; \theta=0 ; h=1$ and boundary conditions at $y=0 ; u=0 ; v=0 ; \theta=0$. On the free surface, $y=h$, the viscous shear stress is balanced by the following gradient of surface tension: $\partial u / \partial y+\partial \theta / \partial x$ $=0$. Scaling and nondimensional parameters are as follows: $x=X / L, \quad y=Y / d, \quad h=H / d, \quad \mathrm{AR}=d / L$; velocity scale $U_{s} \equiv[d \sigma / d T] \mathrm{AR}\left(T_{h}-T_{c}\right) / \mu, u=U / U_{s}, v=\left(V / U_{s}\right) \mathrm{AR}$, $\theta=\left(T-T_{c}\right) /\left(T_{h}-T_{c}\right), \quad p=\left[P-P_{\mathrm{atm}}-\rho g(H-Y)\right] /\left(\mu U_{s} L / d^{2}\right)$, Reynolds number $\operatorname{Re}=U_{s} L / \nu$, Prandtl number $\operatorname{Pr}=\nu / \alpha$, Marangoni number $\mathrm{Ma}=\mathrm{Re} \cdot \mathrm{Pr}$, Bond number $\mathrm{Bo}$ $=\rho g L^{2}[d \sigma / d T] /\left(T_{h}-T_{c}\right)$, and the time scale $\tau$, can be chosen $\tau_{1}=d^{2} / \nu$ (viscous diffusion of momentum across the depth) or $\tau_{2}=L / U_{s}$ (advection across the length). In addition, at $y=h$ it is required that velocity normal to the free surface be zero. This is achieved by restricting the surface height variations through conserving the parameter $\Phi_{H}=Y-H(x, t)$; $D \Phi_{H} / D t=0$ leads to: (a)

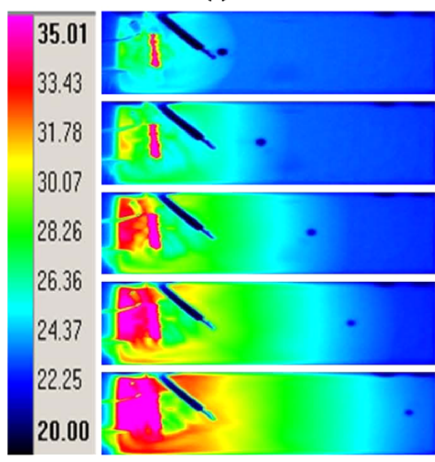

(b)

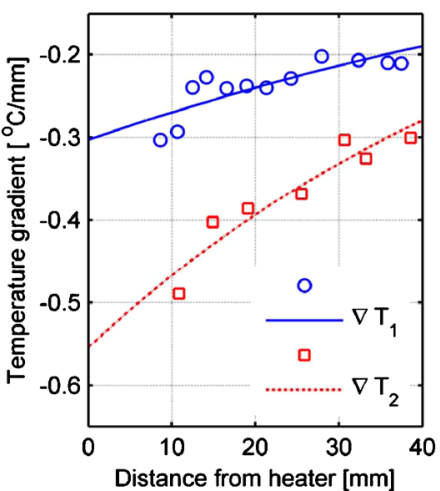

(c)

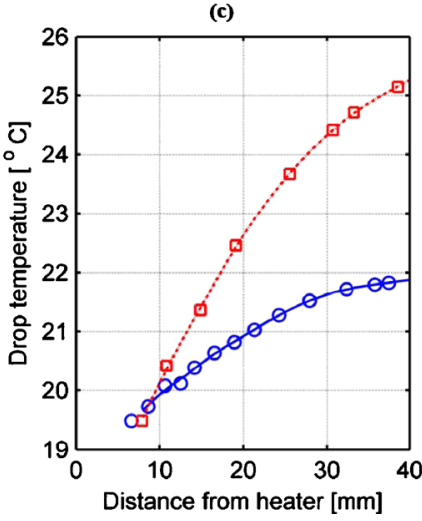

(d)

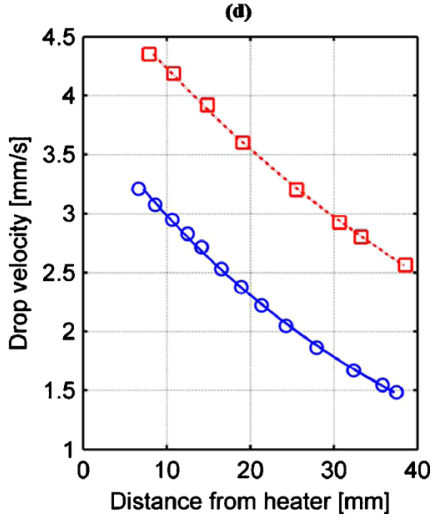

FIG. 3. (Color online) Characteristics of thermocapillary migration of lens-shaped drops away from the heat source $\left(\square: U_{s}=12 \mathrm{~mm} / \mathrm{s}, \mathrm{Re}=230, \mathrm{Ca}=4\right.$ $\times 10^{-3}, \mathrm{Ma}=2 \times 10^{4}$, and $\mathrm{Bo}=3.2 \times 10^{4} ; \mathrm{O}: U_{s}=8.2 \mathrm{~mm} / \mathrm{s}, \mathrm{Re}=160, \mathrm{Ca}=2.7 \times 10^{-3}, \mathrm{Ma}=1.3 \times 10^{4}$, and Bo=5 $\left.\times 10^{4}\right)$ (Video 1 [URL: http://dx.doi.org/ 10.1063/1.3456391.1], enhanced online). 


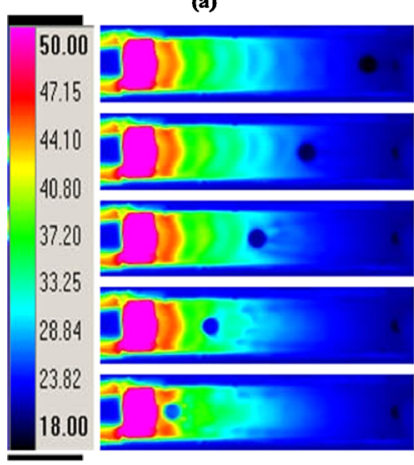

(b)

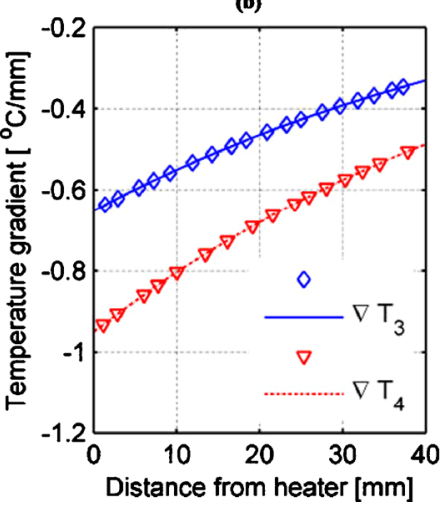

(c)

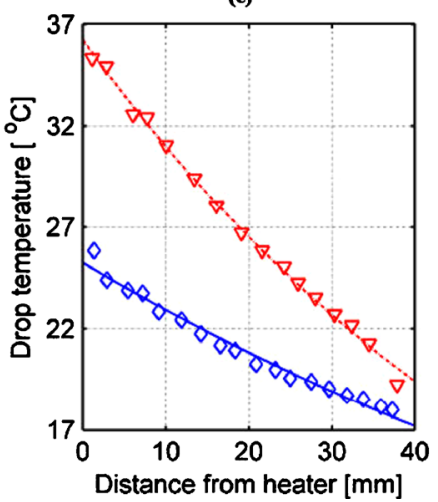

(d)

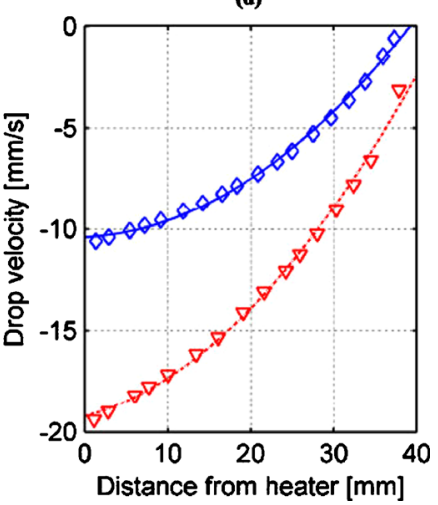

FIG. 4. (Color online) Characteristics of thermocapillary and gravity driven migration of spherical drops toward the source of heat $\left(\nabla: U_{s}=20 \mathrm{~mm} / \mathrm{s}\right.$, Re $=390, \mathrm{Ca}=6.5 \times 10^{-3}, \mathrm{Ma}=3.3 \times 10^{4}$, and $\mathrm{Bo}=1.9 \times 10^{4} ; \diamond: U_{s}=15 \mathrm{~mm} / \mathrm{s}, \mathrm{Re}=300, \mathrm{Ca}=4.9 \times 10^{-3}, \mathrm{Ma}=2.5 \times 10^{4}$, and $\left.\mathrm{Bo}=2.6 \times 10^{4}\right)(\mathrm{Video} 2[\mathrm{URL}$ : http://dx.doi.org/10.1063/1.3456391.2], enhanced online).

$$
u \frac{\partial h}{\partial x}+\left(L / U_{s} \tau\right) \frac{\partial h}{\partial t}=v_{@ y=h} .
$$

By integrating the continuity equation from $y=0$ to the free surface and considering $v_{@ y=0}=0$ then $v_{@ y=h}$ can be replaced in Eq. (4) as follows:

$$
\frac{\partial}{\partial x}\left(\int_{0}^{h(x)} u d y\right)+\left(L / U_{s} \tau\right) \frac{\partial h}{\partial t}=0
$$

Solution to Eqs. (1)-(5) provides the two key results that can be used to explain why the lens and spherical drops move in opposite directions when a thermal gradient is maintained across the interface. (i) On the free interface, there is an outward flow which goes from hot to the cold end. (ii) The interface deforms such that the region close to the hot boundary becomes depressed. The theoretically obtained free surface velocity due to thermocapillary motion is compared with the lens-shaped drop velocity being carried away by the free surface in Fig. 5(a). The normalized velocities are comparable for the two gradients.

In the case of the spherical drop, although the motion is against the direction of the surface flow and is counterintuitive at the outset, a simple analysis can show that spherical drops should indeed roll down the free surface slope due to gravity. The drop acceleration can be attributed to the free surface slope, $H_{x}(x)=d H / d X=\tan (\zeta) \sim \zeta \sim \sin \zeta$ due to small slopes $(\zeta$ is the angle the free surface makes with the horizon). Drop motion is affected by gravity and the opposing
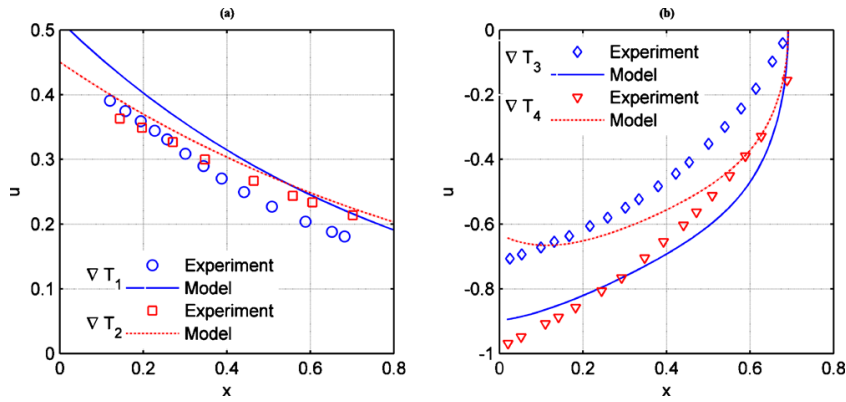

FIG. 5. (Color online) Comparison of normalized velocity from experiment and theory for (a) partially submerged lens drops and (b) spherical drops. viscous drag of the underlying medium as follows: $m a=$ $-m g \sin \xi+C_{D}(1 / 2 \rho A) V_{\text {rel }}^{2}$; using $a \cdot d X=V \cdot d V$ the differential equation governing the drop velocity is

$$
\frac{d V}{d X}=-g \cdot H_{x} \frac{1}{V}+C_{0} \frac{\left(V+V_{\text {surf }}\right)^{2}}{V},
$$

where $C_{0} \equiv C_{D} / m(1 / 2 \rho A)$ and $g$ is the gravitational acceleration and $C_{D}$ is the drag coefficient. There is viscous interaction at the interface of the drop with the surrounding liquid due to the thermally induced interfacial tension gradient. A nominal value for the drag coefficient of 0.5 is used for the sphere whose one-third volume is immersed in the liquid, accounting for the appropriate frontal area $A$. In addition, since the carrier liquid has lower thermal conductivity than the drop (Table I), the drop is essentially isothermal, ruling out the possibility that the surface tension forces at the interface of drop/carrier liquid are responsible for drop migration. Normalized drop velocity obtained from the numerical solution of Eq. (6) predicts the experimental data well for the two temperature gradients, partially validating the ad hoc drag coefficient value. The predictive capability of the model for different values of drag coefficient was marginal, however, it still predicted the direction of the drop motion correctly.

In conclusion, due to a temperature gradient on a thin liquid film, the dual motion of a droplet is observed depending on its shape. This study provides further physical insight into liquid-buffer droplet-based biochemical microsystems and laboratory on chip applications.

${ }^{1}$ A. A. Darhuber, J. P. Valentino, S. M. Troian, and S. Wagner, J. Microelectromech. Syst. 12, 873 (2003).

${ }^{2}$ T. S. Sammarco and M. A. Burns, AIChE J. 45, 350 (1999).

${ }^{3}$ F. Brochard, Langmuir 5, 432 (1989).

${ }^{4}$ J. B. Brzoska, F. Brochard-Wyart, and F. Rondelez, Langmuir 9, 2220 (1993).

${ }^{5}$ R. O. Grigoriev, Phys. Fluids 17, 033601 (2005).

${ }^{6}$ R. O. Grigoriev, M. F. Schatz, and V. Sharma, Lab Chip 6, 1369 (2006).

${ }^{7}$ A. Basu and Y. B. Gianchandani, J. Micromech. Microeng. 18, 115031 (2008).

${ }^{8}$ S. T. Thoroddsen, T. G. Etoh, and K. Takehara, J. Fluid Mech. 478, 125 (2003).

${ }^{9}$ R. Savino, D. Paterna, and M. Lappa, J. Fluid Mech. 479, 307 (2003).

${ }^{10}$ E. F. Greco and R. O. Grigoriev, Phys. Fluids 21, 042105 (2009).

${ }^{11}$ S. M. Pimputkar and S. Ostrach, Phys. Fluids 23, 1281 (1980)

${ }^{12}$ A. K. Sen and S. H. Davis, J. Fluid Mech. 121, 163 (1982). 